\title{
ARTICLE OPEN \\ Surface oxidation mechanism of a refractory high-entropy alloy
}

\author{
Eric Osei-Agyemang ${ }^{1}$ and Ganesh Balasubramanian ${ }^{1}$
}

High-entropy alloys (HEAs) synthesized using refractory elements are being strongly considered as candidates for high temperature structural applications. The role of compositional changes of HEA surfaces due to oxidation is crucial to sustain the material properties, but a detailed description of the thermodynamic mechanism driving the adsorption of oxygen on such complex surfaces is absent. We examine and explain the reaction process of oxygen on a representative refractory HEA surface using first principles and atomistic thermodynamic models. The HEA surface is highly reactive to oxygen yielding a full monolayer coverage at temperatures between 300 and $1500 \mathrm{~K}$. The preferential adsorption of oxygen to specific sites of the HEA surface is attributed to the electronic configuration of the bonding shells of the constituent surface atoms. On further oxygen addition, the oxygen atoms diffuse into the bulk regions of the alloy. Manipulation of temperature and oxygen pressure reveals that it is difficult to rid the alloy surface of oxygen even at extremely low pressures of $10^{-9}$ bar at $2000 \mathrm{~K}$.

npj Materials Degradation (2019)3:20; https://doi.org/10.1038/s41529-019-0082-5

\section{INTRODUCTION}

High-entropy alloys (HEAs), a subset of multi-principal element alloys (MPEAs), contain $N$ principal elements typically in near equiatomic concentrations with $N \geq 5$. ${ }^{1}$ For the majority of the HEAs investigated to date, the predominant phases formed are disordered solid solutions primarily as face-centered cubic (FCC) or body-centered cubic (BCC) crystals, and some hexagonal-closed packed (HPC) phases. These disordered phases are favored over the ordered phases due to many competing parameters such as the enthalpy of mixing, the valence electron concentration, atomic radii, lattice structure and electronegativity of the constituent elements as well as the high ideal configurational entropy of mixing, $\Delta S_{\text {conf }}=-R \Sigma X_{i} \ln \left(X_{i}\right)$ that is equal to or greater than $1.5 \mathrm{R}^{2,3}$ where $X_{i}$ represents the atomic fraction of element $i$ and $R$ is the molar gas constant. This high entropy of mixing reduces the driving forces for the formation of intermetallic phases. ${ }^{4}$ However, the intermetallic phases formed in some cases are due to a considerable atomic size mismatch and large negative enthalpies of mixing of the alloying elements. ${ }^{5,6}$ Ordered solid-solutions have, nevertheless, been observed to exist in some HEAs though they are typically less stable, which affects their properties and microstructures, e.g., through increase in density, higher melting temperatures and increased brittleness of the resulting alloys. ${ }^{3,7-9}$

New structural alloys that can survive extreme temperatures with good oxidation resistance and mechanical properties at elevated temperatures are required in applications such as highspeed turbines and in the aerospace industry. Refractory HEAs offer promising prospects for such applications. Several potential refractory $\mathrm{HEAs}^{7,10-14}$ have been recently identified based on contemporary approaches that involve manipulation of valence electron concentrations, entropy of mixing, the Hume-Rothery rules among others. ${ }^{1,15,16}$ These HEAs have been reported to provide promising alternatives to Ni-based superalloys. Typically, these alloys are composed of three or less phases, with the BCC phase being predominant as the primary phase. ${ }^{4}$ Limited literature on the degradation mechanisms of some HEA surfaces due to oxidation and other physical effects are available, ${ }^{17}$ while the corrosion resistance of several HEAs has been determined both experimentally and computationally primarily focusing on anodic and cathodic reactions. ${ }^{8,19}$ However, to the best of our knowledge, this report is the first study that describes the fundamental thermodynamic mechanisms analyzing how oxygen adsorbs on complex surfaces, such as those of HEAs.

Recently, Singh et al. ${ }^{20}$ proposed refractory Mo-W-Ta-Ti-Zr HEAs with compositions that exhibited greatly enhanced modulus of elasticity $(3 \times$ at $300 \mathrm{~K})$ over the near equiatomic counterparts. Two compositions were found to exhibit mechanical properties better than commercial Mo-based alloys (such as TZM) with a $2.3 \times$ higher modulus of elasticity at $2000 \mathrm{~K}:\left(\mathrm{Mo}_{1-z} \mathrm{~W}_{z}\right)_{0.85} \mathrm{Ta}_{0.10}(\mathrm{TiZr})_{0.05}$ where the alloy identified as $\mathrm{c} 10$ (here referred to as HEA1) has $z=0.5$ and another alloy denoted as $c \overline{10}$ has $z=0.05$ (denoted here as HEA2). The 0.85 signifies that Mo and $W$ together occupy $85 \%$ by mole fraction of the alloy. The $z$ value of 0.5 indicates each of Mo and W is 0.425 mole fraction in that HEA, while $z=0.05$ indicates $M o$ is 0.8075 and $W$ is 0.0425 by mole fraction in the corresponding alloy. As these refractory alloys are potentially useful for operations in high temperature environments, knowledge of their oxidation behavior is required to develop oxidationresistant HEAs. The data on the oxidation mechanisms of HEAs is sparse, and more so for the Mo-W-Ta-Ti-Zr refractory HEA.

Here, we employ density functional theory (DFT) calculations and thermodynamic modeling to understand and explain the oxidation behavior of HEA1 and HEA2 alloys in oxidizing environments. The special quasi-random structures (SQS) method is used to generate random solid solutions for the chosen HEA1 and HEA2 compositions. Different surface models are generated from the constructed random solid solution and the model with the lowest surface energy used. Sequential adsorption of oxygen

\footnotetext{
${ }^{1}$ Department of Mechanical Engineering and Mechanics, Lehigh University, 19 Memorial Drive West, Bethlehem, PA 18015, USA
}

Correspondence: Ganesh Balasubramanian (bganesh@lehigh.edu)

Received: 24 December 2018 Accepted: 23 April 2019

Published online: 09 May 2019 

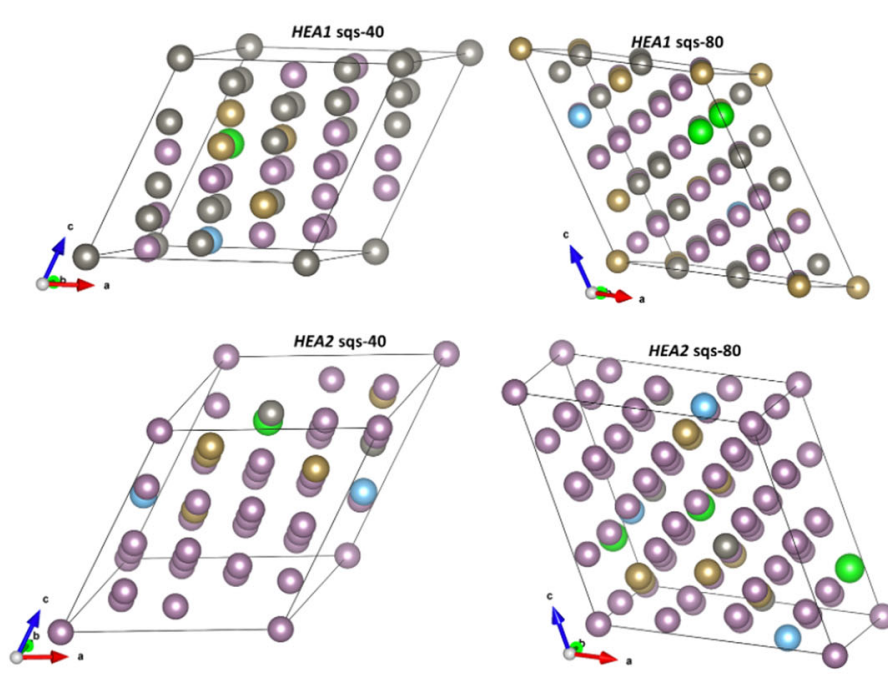

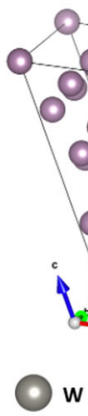

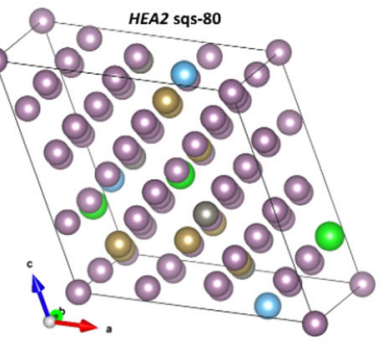

zr

Fig. 1 The generated SQS-N structures for HEA1 and HEA2 alloys

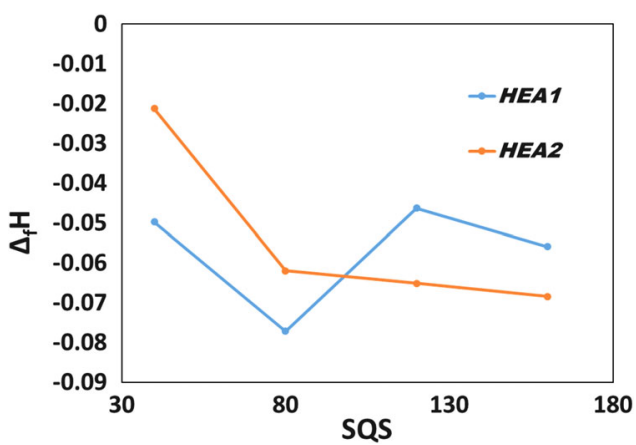

Fig. 2 The calculated enthalpy of formation for the SQS-N structures of HEA 1 and HEA2 alloys. The negative enthalpy implies that clustering/segregation do not occur in these alloys

on the alloy surface is carried out and characterized by the Gibbs free energy of adsorption until full coverage of the surface is obtained. Subsequently, we examine the diffusion of oxygen into the bulk regions of these alloys.

\section{RESULTS AND DISCUSSION}

\section{Alloy structure properties}

Upon relaxing the unit cell of the generated SQS structures, we note that the changes in cell vectors relative to the ideal unrelaxed SQS structures are minimal indicating stability of the produced random solid solution. The pair correlation functions for the alloys are reproduced perfectly for the SQS-40, SQS-80, SQS-120, and SQS-160 structures, where SQS-N denotes structures consisting of $N$ atoms. All the generated structures are monoclinic. Figure 1 shows the ideal unrelaxed structures created using the mcsqs code.

The calculated formation enthalpies for all the generated SQS-N structures are presented in Fig. 2. As the modeled structures contain considerable number of atoms, the calculated formation enthalpies are all negative or closer to zero highlighting the stability in the generated structures. Thus, we do not expect clustering or segregation of the atoms in either the HEA1 or the HEA2 alloy.

The lattice parameter $a$ calculated for all the SQS-N structures for both HEA1 and HEA2 alloys are listed in Table 1. The estimated
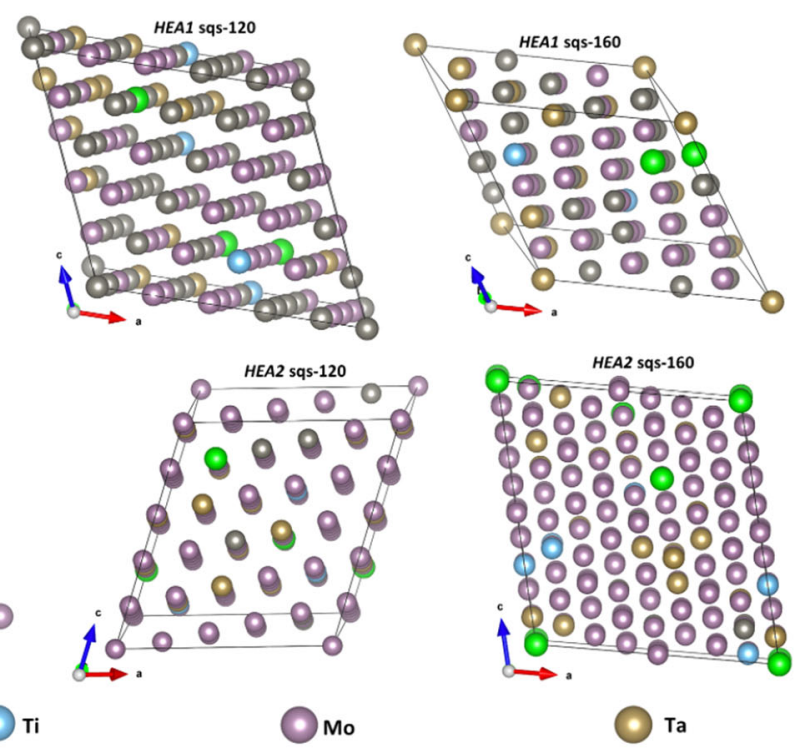

Table 1. Calculated lattice parameter for generated SQS-N structures

\begin{tabular}{llr}
\hline Lattice a $(\AA)$ & HEA1 & HEA2 \\
\hline SQS-40 & 3.215 & 3.241 \\
SQS-80 & 3.194 & 3.189 \\
SQS-120 & 3.191 & 3.177 \\
SQS-160 & 3.186 & 3.179
\end{tabular}

lattice parameters decrease as the SQS size increases with convergence achieved when 80 or more atoms are used in generating the random solid solution. The calculated values are in good agreement with the computed value of $3.171 \AA$ by Singh et al. ${ }^{20}$ using Korringa-Kohn-Rostoker Coherent-Potential Approximation. As the calculated lattice parameter converges at SQS-120, we used the 120-atom structure for further calculations. This selection also ensures that we have a good representation of all atoms on the surface slab for the oxidation studies due to the low concentration of $\mathrm{Ti}$ and $\mathrm{Zr}$ atoms in the alloys.

The total (TDOS) and projected (PDOS) density of states for SQS120 are illustrated in Fig. 3. The Fermi level lies in the pseudo-gap of HEA1, which reflects the stable nature of the bulk alloy obtained through SQS. However, from the DOS, we note that a considerable number of states are present at the Fermi level, most of which are contributed by Mo and $\mathrm{W}-d$ states due to the high relative concentrations of Mo and $W$ atoms in the alloy. Additionally, there are contributions from $\mathrm{Mo}-p, \mathrm{Ta}-d, \mathrm{~W}-p$ states at the Fermi level. These predictions suggest that Mo and $W$ atoms are reactive towards oxygen upon adsorption due to the higher percentage of these atoms compared to the other elements. Thus, the presence of more Mo and $\mathrm{W}$ active sites compared to $\mathrm{Ta}$, $\mathrm{Ti}$ and $\mathrm{Zr}$ atoms will lead to higher oxygen coverage at Mo and $\mathrm{W}$ rich regions.

Generation of appropriate surface structures

The calculated cleavage energies for the (001), (110), and (111) surfaces of HEA1 are listed in Table 2. The (001) face that has the lowest surface energy is selected for the oxidation studies. Due to the random nature of the alloy structure, different surface terminations are possible and hence surface energies are calculated for the different (001) terminations (Table 2). The 


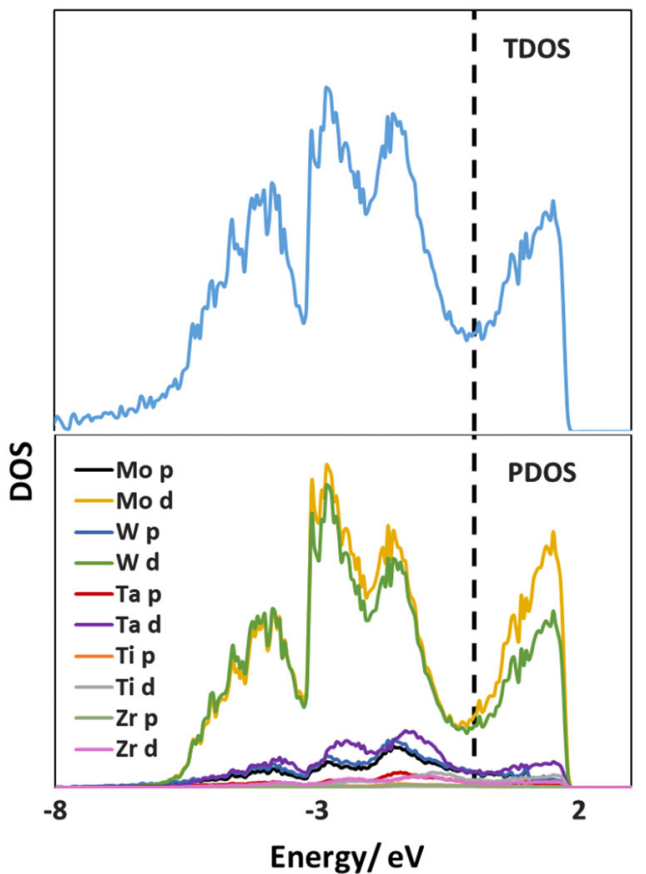

Fig. 3 Total (top) and projected (bottom) density of states (DOS) for bulk HEA 1 alloy suggest that Mo and $\mathrm{W}-d$ states predominantly contribute to the energy states at the Fermi level, implying that the atoms of these elements are reactive to the adsorbed oxygen

Table 2. Cleavage and surface energies for the HEA1 SQS-120 structure

\begin{tabular}{ll}
\hline Surface & Cleavage Energy/ meV. $\AA^{-2}$ \\
\hline (001) & 180.7 \\
$(110)$ & 222.7 \\
$(111)$ & 217.9 \\
\hline HEA1 (001) Surface terminations & Surface Energy/ meV. $\AA^{-2}$ \\
\hline I & 157.2 \\
II & 190.1 \\
III & 182.8 \\
IV & 199.1 \\
\hline
\end{tabular}

different terminations termed as surfaces I, II, III, and IV are presented in supplementary Fig. S1.

All four surfaces yield lower surface energies than the (110) and (111) surfaces, while surface I assumes the lowest. Note that the unit for surface energies is $m e V . \AA^{-2}$, and hence the effective difference in stability between the four surfaces is minimal. In order to observe the effect of oxygen on all the constituent atoms of the HEA, surface termination IV that contains all five atoms of the alloy is used for the adsorption studies. As the difference between HEA1 and HEA2 is the relative fraction of Mo and W atoms (the total number of Mo and $W$ atoms remain the same in both alloys), we assume that the compositions of the most stable HEA1 and HEA2 (001) surfaces are similar (minor difference in the number of Mo and $W$ atoms) and the difference in their surface energies is negligible (in meV. $\AA^{-2}$ ). Hence, the subsequent oxidation studies are performed on HEA1 with similar observations expected for HEA2.
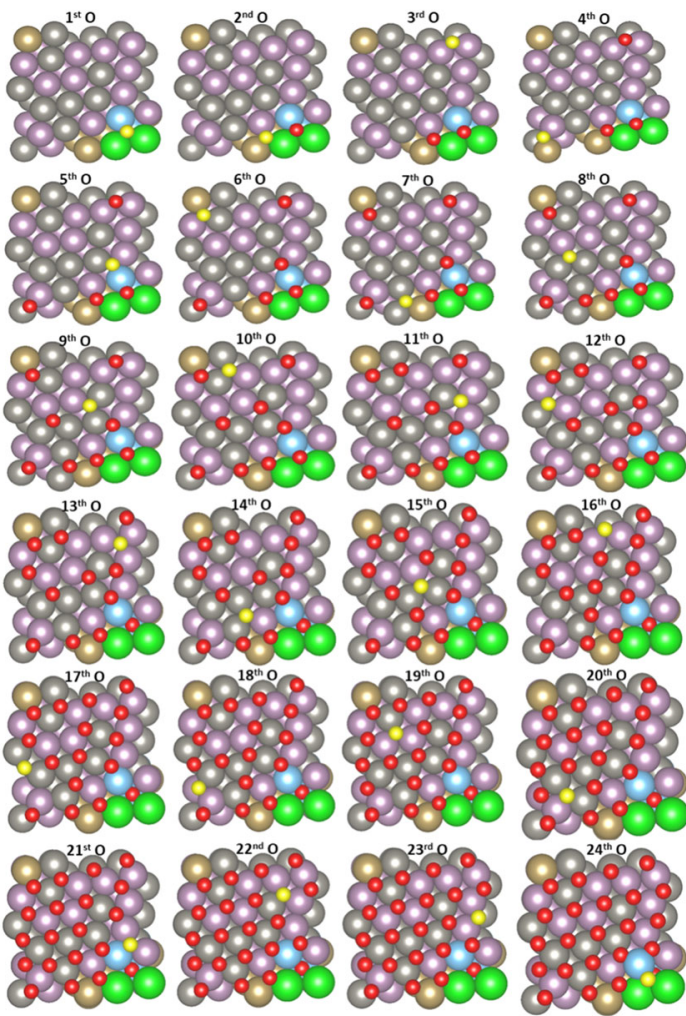

$\mathrm{Zr}$

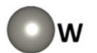

(0)

i Mo
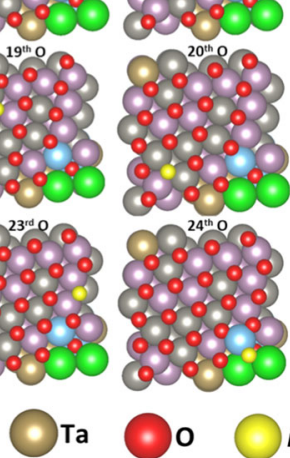

Fig. 4 Top views of oxygen adsorbed (1 to $24 \mathrm{O}$ atoms) HEA1 (001) surfaces. Yellow spheres (ith $\mathrm{O}$ ) represent the currently adsorbed $\mathrm{O}$ atom for the corresponding coverage

Sequential oxidation of the (001) surface

While oxygen is adsorbed on the (001) surface of HEA1 in both associative $\left(\mathrm{O}_{2}\right)$ and dissociative $(\mathrm{O})$ modes, our results suggest a preference for dissociative adsorption of oxygen atoms. A strong interaction of the first $\mathrm{O}$ atom with the surface is characterized by a high adsorption energy. The $\mathrm{O}$ atoms show preferential bonding at the threefold fcc hollow sites between three surface metal atoms. The first $\mathrm{O}$ atom adsorbs to two $\mathrm{Zr}$ and one $\mathrm{Ti}$ surface atoms. The calculated bond distances between the $\mathrm{O}$ atom and the two $\mathrm{Zr}$ atoms are $2.108 \AA$ and $2.175 \AA$, while that between the $\mathrm{O}$ and $\mathrm{Ti}$ atoms is $1.960 \AA$. Figure 4 illustrates the adsorption of oxygen on the HEA1 (001) surface at various coverages.

The calculated adsorption energy $\left(E_{\mathrm{ads}}\right)$ is $5.45 \mathrm{eV}$. An earlier investigation on oxidation of $\mathrm{ZrC}$ found $\mathrm{O}$ to adsorb strongly at $\mathrm{fcc}$ three-fold hollow sites mostly between surface $\mathrm{Zr}$ atoms with $E_{\mathrm{ads}}=5.83 \mathrm{eV}$ on the (111) surface that primarily had only $\mathrm{Zr}$ atoms. $^{21}$ The high adsorption energy predicted from our computations indicates a high reactivity of oxygen towards the HEA1 alloy surface.

We find that the first four added oxygen atoms prefer to bond at sites with available $\mathrm{Zr}$ atoms with successive adsorption energies of 5.45, 5.01, 4.84 and $5.10 \mathrm{eV}$ respectively. The first $\mathrm{O}$ atom with the highest $E_{a d s}$ involves $\mathrm{Zr}$ and $\mathrm{Ti}$ while the next two highest $E_{\mathrm{ads}}(5.01$ and $5.10 \mathrm{eV}$ ) involve $\mathrm{Zr}$ and Ta atoms. The lowest $E_{\text {ads }}$ of $4.84 \mathrm{eV}$ involves $\mathrm{Zr}$ with two Mo atoms. Thus, a high affinity of oxygen to both $\mathrm{Zr}$ atoms is noted followed by $\mathrm{Ti}$, Ta, and $\mathrm{Mo}$ though the percentage of $\mathrm{Zr}, \mathrm{Ti}$, and $\mathrm{Ta}$ active sites are lesser than the Mo and $W$ active sites present on the surface.

Both $\mathrm{Zr}$ and $\mathrm{Ti}$ have the highest number of unfilled $d$ - orbitals (five unfilled $d$ - orbitals), including two partially filled orbitals compared to Mo, W, Ta. This electronic structure results in the 
formation of strong covalent bonds with the adsorbed $\mathrm{O}$ atoms. In supplementary Fig. S4 showing the projected DOS for $\mathrm{Zr}$ and $\mathrm{Ti}$, there are significant conduction band $d$ - states (for $\mathrm{Zr}$ and $\mathrm{Ti}$ ) around the Fermi level that gets filled upon oxygen adsorption.

As the available $\mathrm{Zr}$ sites are saturated the fifth $\mathrm{O}$ atom prefers the available Ti sites with a high successive $E_{\text {ads }}$ of $4.80 \mathrm{eV}$. The bonding here is between the available $\mathrm{Ti}$ site with Mo and $\mathrm{W}$ atoms. As $\mathrm{Ta}$ is the next preferable site after $\mathrm{Zr}$ and $\mathrm{Ti}$, the sixth $\mathrm{O}$ atom bonds at a fcc hollow site between $\mathrm{Ta}, \mathrm{W}$ and $\mathrm{Mo}$ atoms with an $E_{a d s}$ of $4.57 \mathrm{eV}$, lower than those involving $\mathrm{Zr}$ and Ti atoms. The next available Ta site accommodates the seventh $\mathrm{O}$ atom at a site involving two $\mathrm{W}$ atoms with an $E_{\mathrm{ads}}=4.43 \mathrm{eV}$. The next three $\mathrm{O}$ atoms (8th, 9th, and 10th) all adsorb at sites with two $\mathrm{W}$ and one Mo atom with successive $E_{\text {ads }}$ of $4.30,4.18$ and $4.10 \mathrm{eV}$ respectively. The following two $\mathrm{O}$ atoms (11th and 12th) adsorb at hollow sites involving two Mo and one W atoms with $E_{\text {ads }}$ of 4.17 and $4.01 \mathrm{eV}$ respectively. The lower successive adsorption energies as compared to the 8th, 9th, and 10th $\mathrm{O}$ atoms is due to the involvement of more Mo atoms compared to $\mathrm{W}$ atoms. The 13th $\mathrm{O}$ atom occupies a site involving three Mo atoms with a lower $E_{\text {ads }}=3.95 \mathrm{eV}$. Adsorption of the 13 th $\mathrm{O}$ atom causes the $1 \mathrm{st}$ $\mathrm{O}$ atom which initially adsorbed at a three-fold fcc site to shift to a four-fold hollow site involving two $\mathrm{Zr}$, a Ti and an Mo atom. The 14th and 15th $\mathrm{O}$ atoms adsorb at sites involving two $\mathrm{W}$ and one Mo atoms with $E_{\text {ads }}$ of 3.90 and $4.02 \mathrm{eV}$, respectively. As the number of $\mathrm{O}$ atoms on the surface increases, there is lateral repulsion between the oxygen atoms that leads to lower $E_{\text {ads }}$ values. Upon adsorption of the 16th $\mathrm{O}$ atom, there is rearrangement and the adsorbed $\mathrm{O}$ atom sits at a site between a Ta atom and two Mo atoms with $E_{\mathrm{ads}}=4.08 \mathrm{eV}$. The higher adsorption energy is due to the presence of the Ta atom. Adsorption of the 17 th $\mathrm{O}$ atom involves two $\mathrm{W}$ and one Mo atom with $E_{\mathrm{ads}}=3.85 \mathrm{eV}$. There is further rearrangement upon adsorption of the 18th $O$ atom, which is located at a fourfold hollow site between a $\mathrm{Zr}$, two Mo and a W atom with $E_{\text {ads }}=3.78 \mathrm{eV}$. The 19 th $\mathrm{O}$ atom is at a site between two Mo atoms and one $\mathrm{W}$ atom with $E_{\text {ads }}=3.63 \mathrm{eV}$. The 20th $O$ atom adsorbs between two $W$ atoms and one Mo atom with $E_{\mathrm{ads}}=3.11 \mathrm{eV}$. When the $21 \mathrm{st} \mathrm{O}$ atom adsorbs at a site between a Ti atom and two Mo atoms with $E_{\text {ads }}$ of $3.49 \mathrm{eV}$, there is rearrangement of the 5 th $\mathrm{O}$ atom (between $\mathrm{Ti}, \mathrm{W}$, and Mo atoms) as well as the 1st $\mathrm{O}$ atom (which moves further beneath the surface plane by forming a bond with a Ti atom in the second layer). There is further rearrangement when the 22nd $\mathrm{O}$ atom adsorbs between three Mo atoms with $E_{\mathrm{ads}}=3.73 \mathrm{eV}$. The $23 \mathrm{rd}$ $\mathrm{O}$ atom adsorbs between two Mo atoms and one $\mathrm{W}$ atom with $E_{\text {ads }}=3.47 \mathrm{eV}$. Upon adsorption of the 24th $\mathrm{O}$ atom, the $1 \mathrm{st} \mathrm{O}$ atom moves further inwards towards the second atomic layer whiles the 24th atom is located atop the 1 st O atom. Thus, as the full monolayer or ML (here, defined as occupation of all surfaceactive sites) coverage is achieved, oxygen begins to move to the bulk propagating through the region rich in $\mathrm{Zr}$ and $\mathrm{Ti}$ atoms.

The mean adsorption energies $E_{\text {ads }}$ as well as the successive adsorption energies for all other coverages are provided in Table 3. In general, the average adsorption energy as well as the successive adsorption energies for the $O$ atoms decrease with increasing coverage. The strongest $\mathrm{O}$ interactions are observed in $\mathrm{Zr}$ rich regions of the alloy.

The lowest successive adsorption energy of $3.11 \mathrm{eV}$ is noted for the adsorption of the 20th $\mathrm{O}$ atom. This step involves the oxygen atom occupying a three-fold fcc hollow site between a Mo and two W surface atoms. Mo has all $4 d$ orbitals partially filled and energetically stable compared to the other elements, which leads to low affinity for oxygen and results in the lowest successive adsorption energy. Also, it is important to note that W has more $d$ orbitals partially filled than Ta. Thus, the weakest interactions of $O$ atoms are with $\mathrm{W}$ and Mo atoms, but given their higher mole fraction relative to other elements, the corresponding regions contribute to enhanced oxygen coverage on the HEA surface. The
Table 3. The calculated average adsorption energies (Avg. $E_{\text {ads }}$ ) and the successive adsorption energies (Succ. $E_{\text {ads }}$ ) for adsorption of $O$ atoms on the HEA1 (001) surface

\begin{tabular}{llll}
\hline No. of O atoms & Coverage/ML & Avg. $E_{\text {ads }} / \mathrm{eV}$ & Succ. $E_{\text {ads }} / \mathrm{eV}$ \\
\hline 1 & 0.042 & 5.45 & 5.45 \\
2 & 0.083 & 5.23 & 5.01 \\
3 & 0.125 & 5.10 & 4.84 \\
4 & 0.167 & 5.10 & 5.10 \\
5 & 0.208 & 5.04 & 4.80 \\
6 & 0.250 & 4.96 & 4.57 \\
7 & 0.292 & 4.89 & 4.43 \\
8 & 0.333 & 4.81 & 4.30 \\
9 & 0.375 & 4.74 & 4.18 \\
10 & 0.417 & 4.68 & 4.11 \\
11 & 0.458 & 4.63 & 4.17 \\
12 & 0.500 & 4.58 & 4.01 \\
13 & 0.542 & 4.53 & 3.95 \\
14 & 0.583 & 4.49 & 3.90 \\
15 & 0.625 & 4.45 & 4.02 \\
16 & 0.667 & 4.43 & 4.08 \\
17 & 0.708 & 4.40 & 3.85 \\
18 & 0.750 & 4.36 & 3.78 \\
19 & 0.792 & 4.33 & 3.63 \\
20 & 0.833 & 4.26 & 3.11 \\
21 & 0.875 & 4.23 & 3.49 \\
22 & 0.917 & 4.20 & 3.73 \\
23 & 0.958 & 4.17 & 3.47 \\
24 & 1.00 & 4.13 & 3.24 \\
\hline
\end{tabular}

next lowest successive adsorption energy of $3.24 \mathrm{eV}$ is recorded during the adsorption of the 24th $\mathrm{O}$ atom above the site of the $1 \mathrm{st}$ $\mathrm{O}$ atom by pushing the latter into the sub-surface plane. Thus, the initial region at which surface $O$ atoms begin to diffuse from the surface layer into the bulk is the subdomain rich in $\mathrm{Zr}$ and $\mathrm{Ti}$ atoms as shown in Fig. 4.

We further examine the diffusion of oxygen into the bulk structure of the HEA 1 alloy by adding a 25 th $\mathrm{O}$ atom to the fully oxidized surface layer. We observed an exothermic reaction for such process with $E_{\mathrm{ads}}=2.60 \mathrm{eV}$, which is lower than the adsorption energies for all surface processes. We corroborate that complete surface coverage is achieved before diffusion of any oxygen atoms into the bulk structure of the alloy. The 25 th $\mathrm{O}$ atom adsorbs at the region where the 1 st $\mathrm{O}$ atom initially adsorbed. Upon addition of the 25th $\mathrm{O}$ atom, the 1 st $\mathrm{O}$ atom rearranges to occupy a spot on the plane of the first surface layer of atoms (where the 13th $\mathrm{O}$ atom is initially adsorbed). The 25 th $\mathrm{O}$ atom is pushed further down into the inter-planar region between the first and second sub-surface layers and diffuses into the bulk (shown in Fig. 5). This atom sits in the planar region of the second subsurface layer at a hollow site between Mo, W, Ta, Ti atoms and directly atop a Ta atom in the third sub-surface layer.

\section{Atomistic thermodynamic modeling}

We investigate the effects of temperature and pressure on the alloys by thermodynamic modeling using stability plots for the HEA when exposed surfaces are oxidized. The Gibbs free energy of adsorption per surface area is determined as a function of pressure for different temperature regimes. As shown in Fig. 6 for representative oxygen coverages at lower to mid-range 
temperatures $(300-1500 \mathrm{~K})$, the surface is covered with a monolayer of oxygen.

The variations of temperature and pressure are not sufficient to eliminate oxygen from the alloy surface at low to medium temperatures. To remove some of the adsorbed oxygen atoms from the HEA1 surface, a higher temperature of $\sim 2000 \mathrm{~K}$ is selected. The distribution of the Gibbs free energy against pressure at $2000 \mathrm{~K}$ is presented in Fig. 7 (Figure inset shows zoomed-in stability for the $\mathrm{O}_{2}$ pressure region of $10^{-9}-10^{-7}$ bar). As expected at $2000 \mathrm{~K}$, several oxygen atoms can be removed from the surface through pressure variations. Magnified distributions for $\mathrm{O}_{2}$ pressure regions of $10^{-7}-10^{-4}$ bar, and $10^{-4}-10^{2}$ bar are provided as supplementary information (supplementary Figs. S2 and S3 respectively).

Between the pressures of $10^{2}-10^{-4}$ bar, the (001) surface of HEA1 is fully covered (1 ML) with oxygen. As the oxygen pressure is reduced from $10^{-4}$ to $10^{-5}$ bar at $2000 \mathrm{~K}$, there is the removal of one $\mathrm{O}$ atom with the $0.958 \mathrm{ML}$ coverage becoming the most

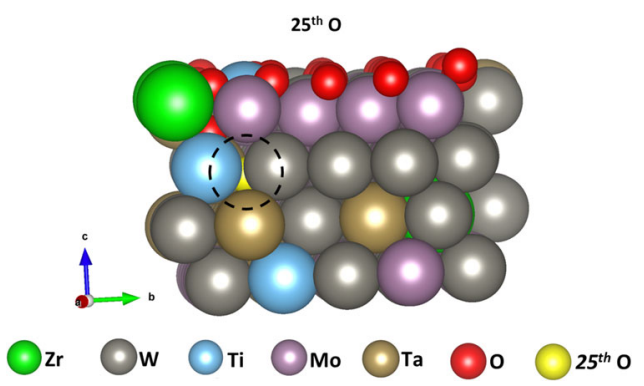

Fig. 5 Side view of the 25th $\mathrm{O}$ atom adsorption on HEA1 (001) surface. Yellow sphere (broken circle area) represents the 25th $\mathrm{O}$ atom in the sub-surface region
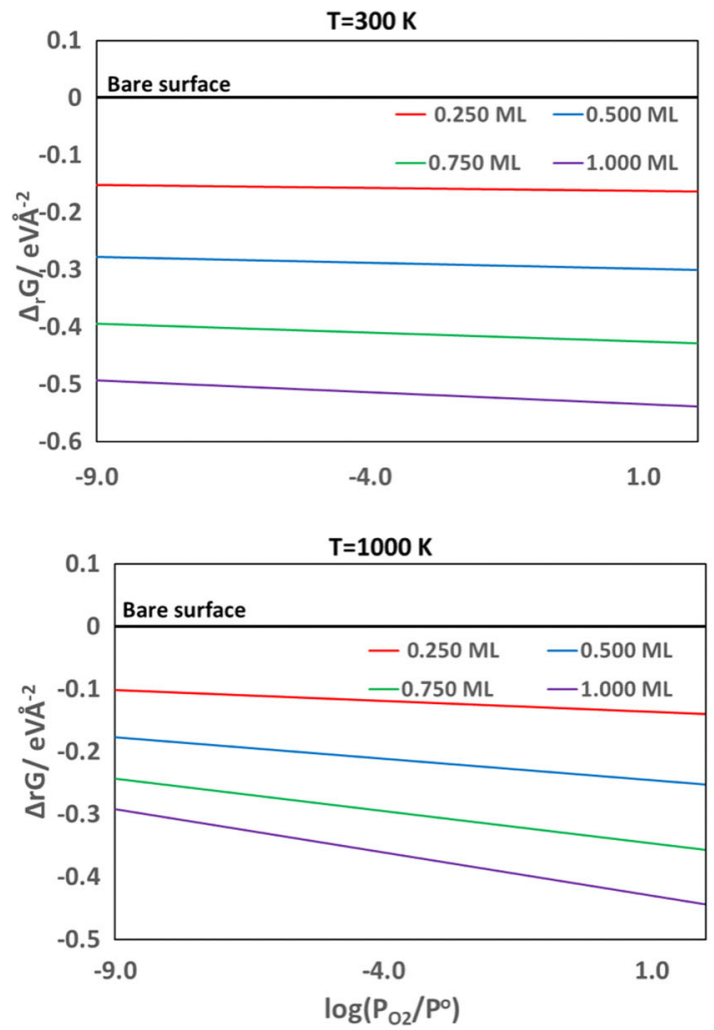

stable. Between $10^{-5}$ and $10^{-5.8}$ bar, there is further removal of four oxygen atoms, yielding a coverage of $0.792 \mathrm{ML}$ on the surface. As the oxygen pressure is further reduced from $10^{-5.8}$ to $10^{-6.6}$ bar, one more oxygen atom is removed from the surface resulting in $0.750 \mathrm{ML}$ coverage. Reducing from $10^{-6.6}$ to $10^{-7} \mathrm{bar}$ of oxygen pressure, there is further removal of one oxygen atom to yield $0.708 \mathrm{ML}$ coverage. With further lower oxygen pressure regimes, another oxygen atom is removed from $10^{-7}$ to $10^{-7.6} \mathrm{bar}$ to yield $0.667 \mathrm{ML}$ coverage. Between $10^{-7.6}$ and $10^{-7.8}$ bar, four oxygen atoms are further removed from the surface, yielding approximately. $0.500 \mathrm{ML}$ coverage. From $10^{-7.8}$ to $10^{-8.4} \mathrm{bar}$ oxygen pressure, another $\mathrm{O}$ atom is eliminated from the surface to yield $0.458 \mathrm{ML}$ coverage, while from $10^{-8.4}$ to $10^{-8.6}$ bar pressure reduction, two oxygen atoms are released from the surface, which yields a $0.375 \mathrm{ML}$ coverage. Between the extremely low oxygen pressures of $10^{-8.6}-10^{-9}$ bar, an additional oxygen atom gets removed to form $0.333 \mathrm{ML}$ coverage. From this analysis, it is evident that even at extremely low oxygen pressures of $10^{-9}$ bar at $2000 \mathrm{~K}$, a significant number of oxygen atoms are present on the surface $(0.333 \mathrm{ML})$. Thus, temperature and pressure variations are not sufficient to retrieve the bare alloy surface once oxidation sets in.

The oxidation process on the (001) surface of the refractory $\left[\left(\mathrm{Mo}_{1-z} \mathrm{~W}_{z}\right)_{0.85} \mathrm{Ta}_{0.10}(\mathrm{TiZr})_{0.05}(z=0.5)\right]$ HEA alloy is examined. The SQS method is used to generate random solid solution structures with the desired composition while surface energies of different terminations are used to select an appropriate alloy surface for oxygen adsorption. Sequential adsorption of oxygen atoms at the available alloy active sites suggests a very strong interaction of the HEA with oxygen. We note a high affinity of oxygen to $\mathrm{Zr}$ atoms followed by Ti, Ta and Mo. The high affinity of oxygen to $\mathrm{Zr}$ and $\mathrm{Ti}$ atoms compared to the other elements is due to the electronic configuration of the bonding shells. A complete coverage with oxygen occurs for the HEA surface as long as there is the
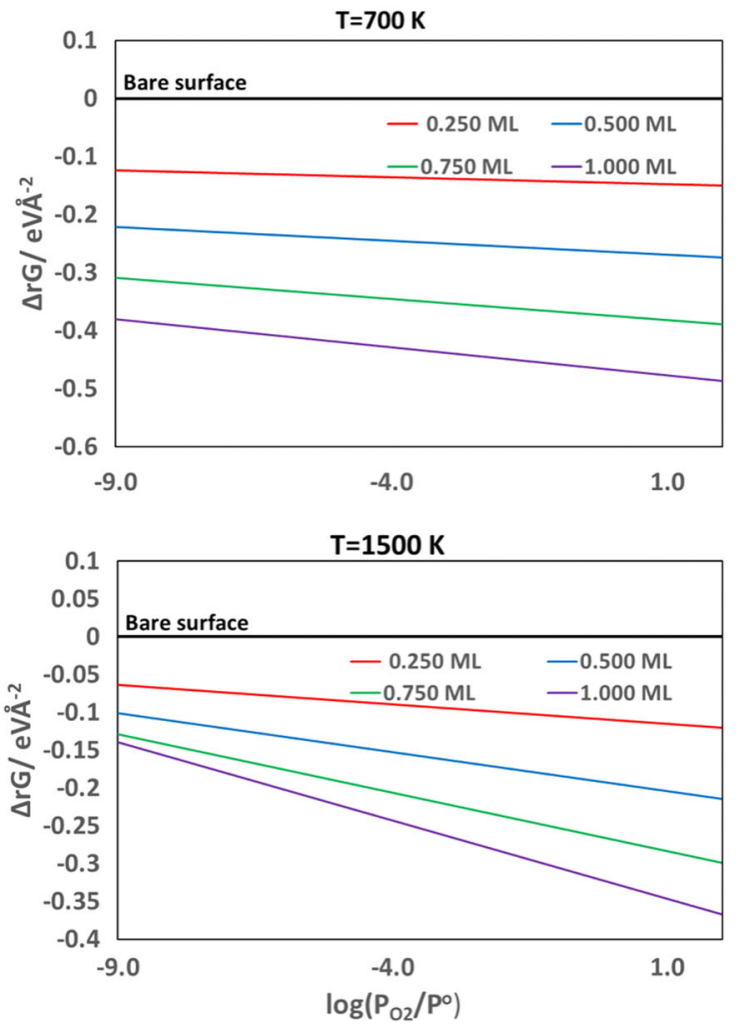

Fig. 6 The variation in the Gibbs free energy with pressure for the adsorption of oxygen atoms on the (001) surface of HEA1 alloy for $300-1500 \mathrm{~K}$ from $10^{-9}$ to $10^{2}$ bar $\mathrm{O}_{2}$ pressure. The distribution shows the HEA1 surface to be completely covered with a monolayer of oxygen at all pressures between 300 and $1500 \mathrm{~K}$ 


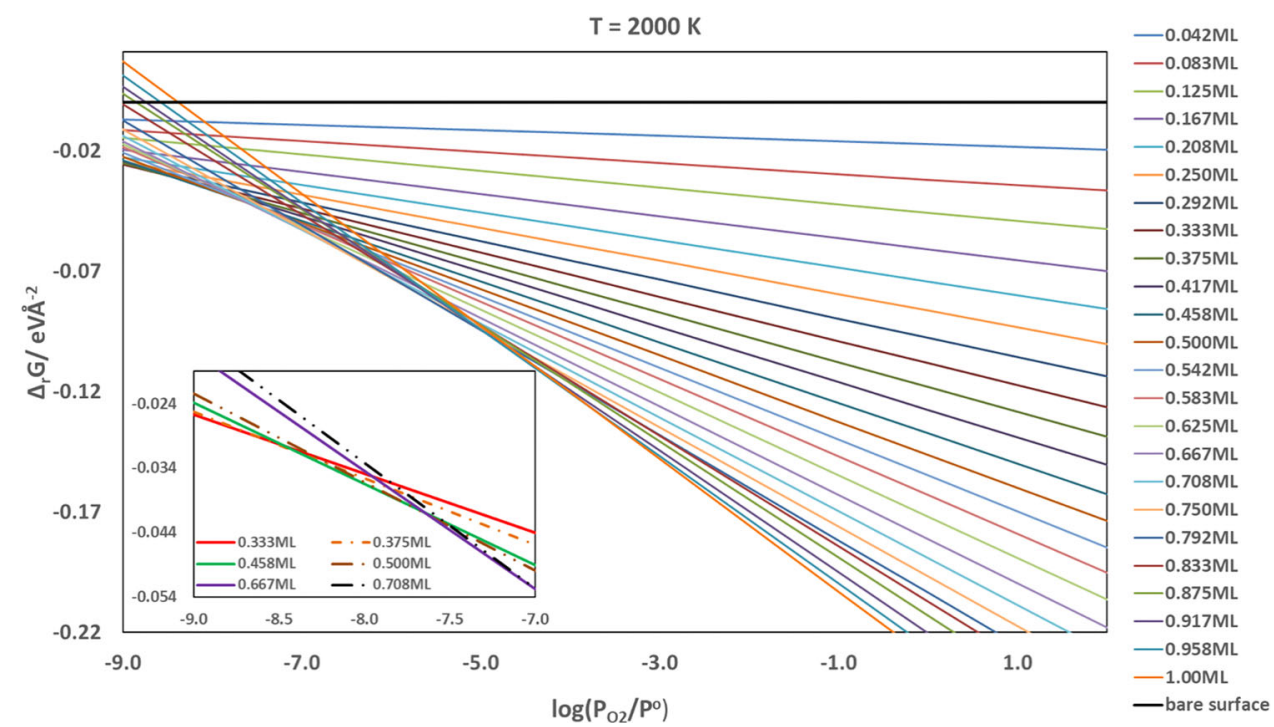

Fig. 7 The variation in the Gibbs free energy with $\mathrm{O}_{2}$ pressure (from $10^{-9}$ to $10^{2}$ bar) for the adsorption of oxygen atoms on the (001) surface of HEA 1 alloy at $2000 \mathrm{~K}$. The inset shows the stability plots within the region of $10^{-9}-10^{-7}$ bar $\mathrm{O}_{2}$ pressure. Stability at different coverages change as the pressure is varied

availability of active sites. Once the exposed surface layer is completely covered with oxygen, further addition of oxygen promotes diffusion of $\mathrm{O}$ atoms into the second and third subsurface layers (bulk regions) of the alloy. As the full monolayer coverage is achieved, oxygen begins to move into the bulk propagating through the region rich in $\mathrm{Zr}$ and $\mathrm{Ti}$ atoms.

We characterize the thermodynamic properties of the oxidation process as a function of temperature and pressure using the Gibbs free energy of adsorption per surface area. We observe that the surface is fully covered (a single monolayer i.e., $1 \mathrm{ML}$ ) with oxygen at temperatures between 300 and $1500 \mathrm{~K}$. At extremely high temperatures $(2000 \mathrm{~K})$ we observe the removal of oxygen atoms with the manipulation of the oxygen pressure. Even at $10^{-9}$ bar, there is still $0.333 \mathrm{ML}$ oxygen coverage on the (001) alloy surface. The bare alloy surface could not be completely recovered through variations of temperature and pressure.

Further efforts are required to understand the effect of different surface compositions with varying concentrations of the refractory elements to analyze the effect of such compositional changes on the oxidation mechanism. Future efforts will also involve understanding the diffusion of oxygen into the bulk alloy and measuring the kinetic rate of the associated processes. Additionally, the effect of oxidation on mechanical properties such as coefficient of thermal expansion will be an area of future investigation.

\section{METHODS}

\section{SQS models}

In order to generate the random solid solutions that represent the HEA1 and HEA2 alloys, the SQS method is employed. For ordered systems, DFT can be used to generate cells with periodic boundary conditions. However, one encounters complications when treating disordered solid solutions for which the use of DFT in generating structures is inappropriate. For fivecomponent HEA1 or HEA2 alloys, one strategy to obtain a random solid solution structure is to construct large supercells where the atoms $A, B, C$, $D$, and $E$ are used to randomly decorate the host lattices. ${ }^{22}$ DFT calculations are however limited by the number of atoms that can be considered in the simulated system and since such large supercells for random solid solutions will involve many atoms, this method is computationally prohibitive. Zunger et al. ${ }^{23-25}$ developed the SQS method to overcome the complexities associated with the large supercells as well as the meanfield theories such as coherent particle approximation (CPA) among others. SQS are built with small-unit-cell periodic structures that mimic the most relevant information such as pair and multisite correlation function of the alloy. ${ }^{22}$ In generating the SQS structure, a distribution of distinct local environments is maintained. The average of those distinct local environments corresponds to the random solid solution. Many important properties of an alloy can be obtained from a single DFT calculation on an SQS structure based on the existence of distinct local environments. The errors introduced by representing random solid solutions with small periodically repeated unit cells can be mitigated by constructing SQS structures to reproduce the correlation functions between the first few nearest neighbors. Also, the errors introduced by periodicity are shifted to the relatively distant neighbors since the near neighbor interactions are more important.

The mcsqs code implemented in the Atom-Theoretic Automated Toolkit (ATAT) $^{26}$ is used to generate various SQS-N structures $(N=40,80,120$, and 160) for the bcc HEA1 and HEA2 random solid solutions. Initially, all structures with $\mathrm{N}$ atoms per unit cell based on the bcc lattice are generated exhaustively with the mcsas code. The pair and multi-site correlation functions for each structure are then constructed, and the structures that best fit the correlation functions of the alloys for specified sets of pair and multisite parameters are explored. The pair correlation functions are constrained to match with those of a random solid solution up to the seventh-nearest neighbor.

\section{DFT calculations}

The Vienna ab initio Simulation Package (VASP) ${ }^{27}$ based on Mermin's finite temperature $\mathrm{DFT}^{28}$ is employed to perform all the calculations. The electronic configurations used to represent the Mo, $\mathrm{W}, \mathrm{Ta}, \mathrm{Ti}, \mathrm{Zr}$, and $\mathrm{O}$ atoms are $[K r] 4 d^{5} 5 s^{1},[X e] 4 f^{14} 5 d^{4} 6 s^{2},[X e] 4 f^{14} 5 d^{3} 6 s^{2},[A r] 3 d^{2} 4 s^{2},[K r] 4 d^{2} 5 s^{2}$, and $[\mathrm{He}] 2 \mathrm{~s}^{2} 2 \mathrm{p}^{4}$ respectively. The Projector Augmented Wavefunction (PAW) pseudopotentials ${ }^{29}$ are used to represent the core electrons as well as the core part of the valence electron wavefunctions that are kept frozen. The PAW representation reduces the number of planewaves required to effectively describe the electrons close to the nuclei. The generalized gradient approximation (GGA) exchange correlation functional as parametrized by Perdew, Burke and Ernzerhof $(\mathrm{PBE})^{30}$ is used while employing the Methfessel-Paxton ${ }^{31}$ smearing scheme by setting the gamma parameter to $0.1 \mathrm{eV}$. An energy cut-off of $600 \mathrm{eV}$ is used for the planewaves expansion. A Monkhorst-Pack ${ }^{32}$ special grid sampling of the k-points for integration of the Brillouin zone yields $4 \times 4 \times 4$ k-points representing 36 irreducible number of sampling points for all bulk calculations and $4 \times 4 \times$ $1 \mathrm{k}$-points for surface calculations. The self-consistent field procedure is used for resolution of the Kohn-Sham equations by setting energy changes for each cycle at $10^{-4} \mathrm{eV}$ as the convergence criterion between two successive iterations. Both volume and shape of the unit cell as well as all the atomic positions for the generated SQS structures are thoroughly relaxed. However, the positions of all the ions in the two top most layers 
are relaxed until the net forces acting on them are smaller than $10^{-2} \mathrm{eV} / \AA$ for all surface calculations.

In order to analyze the phase stability of the HEA1 and HEA2 alloys, the formation enthalpies of the SQS-N structures are calculated using the DFT bulk total energies of the constituent elements in their ground state. Equation 1 is used to calculate the formation enthalpies.

$$
\begin{gathered}
\Delta \mathrm{H}(\mathrm{HEA} 1, \mathrm{HEA} 2)=\frac{1}{(a+b+c+d+e)} \\
{\left[E\left(\mathrm{Mo}_{\mathrm{a}} \mathrm{W}_{\mathrm{b}} \mathrm{Ta}_{\mathrm{c}} \mathrm{Ti}_{\mathrm{d}} \mathrm{Zr}_{\mathrm{e}}\right)-a E(\mathrm{Mo})-b E(\mathrm{~W})-c E(\mathrm{Ta})-d E(\mathrm{Ti})-e E(\mathrm{Zr})\right]}
\end{gathered}
$$

Here, $E(M o), E(\mathrm{~W}), E(T a), E(T i), E(Z r)$ are the DFT total energies of pure bccMo, bcc-W, bcc-Ta, hcp-Ti, and hcp-Zr elements in their ground state structures while $E\left(\mathrm{Mo}_{\mathrm{a}} \mathrm{W}_{\mathrm{b}} \mathrm{Ta}_{\mathrm{c}} \mathrm{Ti}_{\mathrm{d}} \mathrm{Zr}_{\mathrm{e}}\right)$ is the DFT total energy of the relaxed SQS-N structure.

In selecting the most appropriate surface for oxygen adsorption studies, surface energies for the (100), (110), and (111) low index surfaces are calculated and the surface with the lowest energy is selected. As solidsolution HEAs have structures with random distribution of constituent atoms, there are additional challenges in selecting the most stable surface for surface adsorption studies. Different surface layers containing different atomic compositions are generated and their surface energies calculated for the (100), (110), and (111) facets. To study the effect of oxygen on all five constituent elements of the alloy, a surface model containing Mo, W, $\mathrm{Ta}, \mathrm{Ti}$, and $\mathrm{Zr}$ atoms with the lowest surface energy is subsequently generated and employed for the oxidation studies.

Due to the random nature of the HEA, cleavage of the bulk yields two inequivalent surfaces with different compositions. In order to limit the number of surfaces to be dealt with, cleavage energy is computed for the as-cleaved (001), (110), and (111) facets from $E_{\text {cleav }}^{A+B}=$ $\frac{1}{2 A}\left[E_{\text {slab }}^{A}+E_{\text {slab }}^{B}-n E_{\text {bulk }}\right]$ and the surface with the lowest cleavage energy is used for further calculations. Here, $E_{\text {bulk }}$ is the energy per atom in the bulk (formula unit), $E_{\text {slab }}^{i}$ is the slab energy for the $i$ slab termination, $n$ is the number of formula units in the bulk and $A$ is the exposed surface area.

As different surface terminations can be obtained for either the (001), (110) or (111) surfaces, we select the appropriate surface by generating symmetric slabs with similar terminating layers (i) on both sides due to the random nature of the alloy structure. Their surface energies are computed using Eq. 2 as:

$\gamma^{i}=\frac{1}{2 A}\left[E_{\text {slab }}^{i}-m E_{\text {bulk }}\right]$

Here, $E_{\text {slab }}$ represents the total energy of the $i$ terminated symmetric slab, $E_{\text {bulk }}$ is the bulk energy per unit formula of HEA1 or HEA2, $m$ is the total number of bulk formula units in both slabs, and $A$ is the exposed surface area. The surface energy in Eq. 2 is for the rigid surface without any relaxations. For the relaxed surface, the relaxation energy for the slab is added to that of the rigid surface and the surface energy is calculated from Eq. 2.

$(1 \times 1)$ surface unit cells generated from the selected SQS structure are used for all surface calculations. A $15 \AA$ vacuum is included as separation between two periodically repeated slabs to avoid surface-surface interactions. For calculations on adsorption of oxygen on the selected surface, four atomic layers are used. The topmost two layers of the surface slabs are relaxed while the remaining layers are kept fixed to mimic bulk properties.

Eleven Mo atoms, eight $\mathrm{W}$ atoms, two Ta atoms, one Ti atom and two $\mathrm{Zr}$ atoms are exposed on the selected surface as shown in Fig. 8 . The $(1 \times 1)$ supercell used for oxygen adsorption on the selected surface has both $a$ and $b$ parameters equal to $13.1558 \AA$, while $a$ and $\beta$ are $90^{\circ}$ and $r$ is 93.3723 $3^{\circ}$ with an exposed surface area of $172.7754 \AA^{2}$.

Oxygen adsorbs on the surfaces in both associative and dissociative modes, and the adsorption energies are computed from Eq. 3 as:

$E_{\text {ads }}=-\left[E_{\mathrm{O} / \text { surf }}-E_{\text {Surface }}-E_{\mathrm{O} 2}\right]$

$E_{O / S u r f}$ is the energy of the oxidized surface, $E_{\text {Surface }}$ is the energy of the clean surface and $E_{\mathrm{O} 2}$ is the energy of the gas phase $\mathrm{O}_{2}$ molecule. The binding energy for $\mathrm{O}_{2}$ is predicted to be $-6.04 \mathrm{eV}$ which compares well with $-6.08 \mathrm{eV}$ calculated in earlier reports. ${ }^{33}$ The binding energy is overestimated by $0.87 \mathrm{eV}$ as compared to the experimental value of -5.17 $\mathrm{eV}^{34}$ which is typical for GGA DFT calculations. To rectify the $\mathrm{O}_{2}$ overbinding, the adsorption energies are corrected by adding half of the overbinding energy $(\sim 0.44 \mathrm{eV})$ to each $\mathrm{O}$ atom adsorbed.

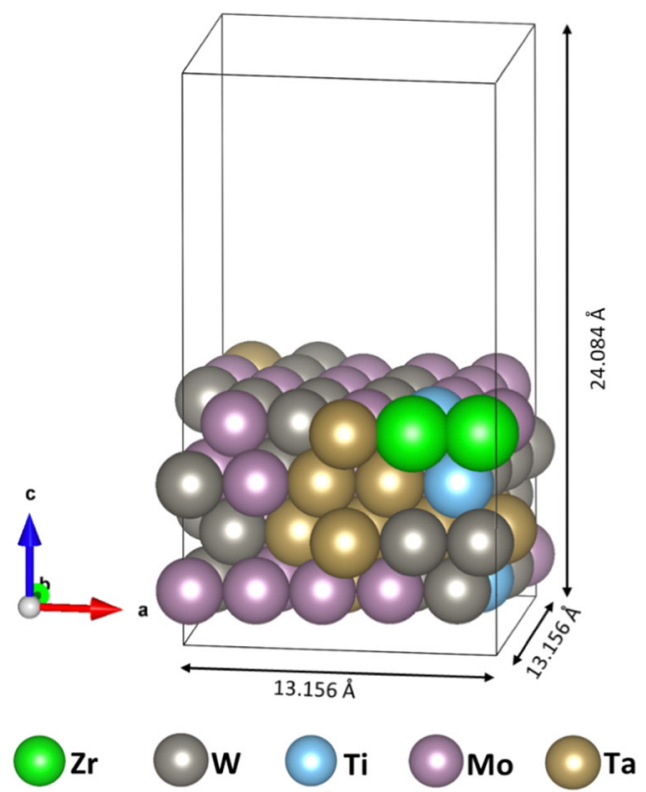

Fig. 8 An atomic representation of the HEA1 (001) surface slab employed for the oxidation calculations

The effect of different coverages of oxygen are evaluated by using the various active metal sites with increasing number of oxygen atoms or molecules at the adsorption sites after identifying the preferred mode of $\mathrm{O}_{2}$ adsorption. As there are 24 active metal sites on the exposed surface, we evaluate 0.042 monolayer (ML), $0.083 \mathrm{ML}, 0.125 \mathrm{ML}$, up to $1 \mathrm{ML}$ full coverage. The number of available metal sites on the exposed surface defines the different coverages. We observe that spin polarized calculations have negligible effect on the $\mathrm{O}_{2}$ adsorption so non-spin polarized calculations are used.

\section{Atomistic thermodynamic model}

We use the well-established thermodynamic model ${ }^{35}$ to provide a relationship between the calculated parameters and experimental working conditions. Here, we assume that the adsorbed atoms or molecules on the surfaces are in thermodynamic equilibrium with the gas phase that serves as a reservoir for gas molecules. This presumption permits for a definition of the Gibbs free energy of adsorption $\left(\Delta_{r} G\right)$ as a function of thermodynamic parameters such as temperature, pressure and chemical potential using Eq. 4:

$$
\Delta_{r} G=\left[\Delta E_{O}+E_{\mathrm{ZPE}(\text { surf } / \mathrm{O} 2)}-\Delta \sum n \mu(T, p)\right]
$$

Here, $E_{Z P E(\text { surface/O2) }}$ is the zero point energy (ZPE) contribution of the $\mathrm{O}_{2}$ molecule when adsorbed on the surface. $\Delta E_{o}$ is the difference in the electronic energies of the oxidized surface and the gaseous $\mathrm{O}_{2}$ molecule as well as any released species, while $\Delta \mu(T, p)$ is the difference in chemical potential of the gas phase $\mathrm{O}_{2}$ molecules and all other released species of the reaction. $\Delta E_{o}$ can be computed according to the surface reaction as described by Eq. 5:

$$
\mathrm{HEA} 1_{\text {surface }}+\mathrm{n}\left(\mathrm{O}_{2}\right) \leftrightarrow \mathrm{HEA} 1_{\text {oxidized-surface }}+\text { released species }
$$

$\Delta E_{o}$ is determined as $\left[E_{\mathrm{el}}\right.$ (oxidized-surface) $+E_{\text {released-specie }}-E_{\mathrm{el}}$ (Surface) $\left.n E_{\mathrm{el}}(\mathrm{O} 2)\right]$. This approach provides the corresponding reaction energies of the small molecules on the oxidized surface. Changes in the chemical potential are brought about by the thermal contributions of the gas phase molecules and it comprises of the temperature dependent terms $\Delta \mu^{\circ}(T)$ and $R T$, as in Eq. 6.

$\Delta \mu(T, P)=\Delta \mu^{o}(T)+R T \ln \left(\frac{P}{P^{\circ}}\right)$

Changes in chemical potential of the gas phase molecules can be calculated using statistical thermodynamics (Eq. 7) as:

$$
\begin{aligned}
\Delta \mu^{\circ}(T)= & {\left[E_{\mathrm{ZPE}}+E_{\mathrm{vib}(0 \rightarrow \mathrm{T})}+E_{\text {rot }}+E_{\text {trans }}\right] } \\
& +R T-T\left(S_{\text {vib }}+S_{\text {rot }}+S_{\text {trans }}\right)
\end{aligned}
$$


Thermal contributions of the small molecules upon adsorption on the surface are estimated from the changes in the vibrational, rotational and translational degrees of freedom. The values of $\Delta \mu^{\circ}(\mathrm{T})$ at different temperatures are computed from standard statistical thermodynamic formulas combined with calculated frequencies from the equilibrium geometry. The total pressure $P^{\circ}$ in Eq. 6 is set at 1 bar. The distribution of the calculated Gibbs free energies $\left(\Delta_{r} G\right)$ as a function of pressure $p$ at different temperatures with the different coverages of $\mathrm{O}_{2}$ molecules exemplify the thermodynamic stability of these surfaces. The Bader code ${ }^{36}$ optimized for VASP is used to analyze the charge densities obtained for both the clean as well as the oxidized surfaces.

\section{DATA AVAILABILITY}

The data that support the findings of this study are available from the corresponding author upon reasonable request.

\section{ACKNOWLEDGEMENTS}

The work was supported by the Office of Naval Research (ONR) through award N00014-18-1-2484. The computations were carried out with the support of Dr. S. Rangarajan and the cluster Sol managed by the Library and Technology Services Research Computing at Lehigh University.

\section{AUTHOR CONTRIBUTIONS}

E.O.-A. performed all the calculations and thermodynamic modeling. G.B. conceived the idea. Both authors contributed to the manuscript preparation and discussion of the results.

\section{ADDITIONAL INFORMATION}

Supplementary information accompanies the paper on the npj Materials Degradation website (https://doi.org/10.1038/s41529-019-0082-5).

Competing interests: The authors declare no competing interests.

Publisher's note: Springer Nature remains neutral with regard to jurisdictional claims in published maps and institutional affiliations.

\section{REFERENCES}

1. Yeh, J.-W. et al. Nanostructured high-entropy alloys with multiple principal elements: novel alloy design concepts and outcomes. Adv. Eng. Mater. 6, 299-303 (2004).

2. Yeh, J.-W. Alloy design strategies and future trends in high-entropy alloys. JOM 65, 1759-1771 (2013).

3. Miracle, D. et al. Exploration and development of high entropy alloys for structural applications. Entropy 16, 494-525 (2014).

4. Senkov, O. et al. Development of a refractory high entropy superalloy. Entropy 18, 102 (2016).

5. Zhang, Y., Yang, X. \& Liaw, P. K. Alloy design and properties optimization of highentropy alloys. JOM 64, 830-838 (2012).

6. Zhang, Y., Zhou, Y. J., Lin, J. P., Chen, G. L. \& Liaw, P. K. Solid-solution phase formation rules for multi-component alloys. Adv. Eng. Mater. 10, 534-538 (2008).

7. Senkov, O. N., Senkova, S. V., Miracle, D. B. \& Woodward, C. Mechanical properties of low-density, refractory multi-principal element alloys of the $\mathrm{Cr}-\mathrm{Nb}-\mathrm{Ti}-\mathrm{V}-\mathrm{Zr}$ system. Mat. Sci. Eng. A-Struct. 565, 51-62 (2013).

8. Wang, Y. P., Li, B. S. \& Fu, H. Z. Solid solution or intermetallics in a high-entropy alloy. Adv. Eng. Mater. 11, 641-644 (2009).

9. Singh, S., Wanderka, N., Murty, B. S., Glatzel, U. \& Banhart, J. Decomposition in multi-component AlCoCrCuFeNi high-entropy alloy. Acta Mater. 59, 182-190 (2011).

10. Senkov, O. N., Wilks, G. B., Miracle, D. B., Chuang, C. P. \& Liaw, P. K. Refractory high-entropy alloys. Intermetallics 18, 1758-1765 (2010).

11. Senkov, O. N., Scott, J. M., Senkova, S. V., Miracle, D. B. \& Woodward, C. F. Microstructure and room temperature properties of a high-entropy TaNbHfZrTi alloy. J. Alloy. Compd. 509, 6043-6048 (2011).

12. Senkov, O. N. et al. Microstructure and elevated temperature properties of a refractory TaNbHfZrTi alloy. J. Mater. Sci. 47, 4062-4074 (2012).
13. Senkov, O. N. \& Woodward, C. F. Microstructure and properties of a refractory NbCrMo0.5Ta0.5TiZr alloy. Mat. Sci. Eng. A-Struct. 529, 311-320 (2011).

14. Senkov, O. N., Senkova, S. V., Dimiduk, D. M., Woodward, C. \& Miracle, D. B. Oxidation behavior of a refractory NbCrMo0.5Ta0.5TiZr alloy. J. Mater. Sci. 47, 6522-6534 (2012).

15. Yeh, J. W. Recent progress in high-entropy alloys. Ann. Chim. Sci. Mat. 31, 633-648 (2006).

16. Yeh, J. W. et al. High-Entropy Alloys - A New Era of Exploitation. Mater. Sci. Forum 560, 1-9 (2007).

17. Ayyagari, A. V., Gwalani, B., Muskeri, S., Mukherjee, S. \& Banerjee, R. Surface degradation mechanisms in precipitation-hardened high-entropy alloys. Npj Mater. Degrad. 2, 33 (2018).

18. Qiu, Y., Thomas, S., Gibson, M. A., Fraser, H. L. \& Birbilis, N. Corrosion of high entropy alloys. Npj Mater. Degrad. 1, 15 (2017).

19. Taylor, C. D., Lu, P., Saal, J., Frankel, G. S. \& Scully, J. R. Integrated computational materials engineering of corrosion resistant alloys. Npj Mater. Degrad. 2, 6 (2018).

20. Singh, P. et al. Design of high-strength refractory complex solid-solution alloys. Npj Comput. Mater. 4, 16 (2018).

21. Osei-Agyemang, E., Paul, J.-F., Lucas, R., Foucaud, S. \& Cristol, S. Oxidation and equilibrium morphology of zirconium carbide low index surfaces using DFT and atomistic thermodynamic modeling. J. Phys. Chem. C. 120, 8759-8771 (2016).

22. Jiang, C., Wolverton, C., Sofo, J., Chen, L.-Q. \& Liu, Z.-K. First-principles study of binary bcc alloys using special quasirandom structures. Phys. Rev. B 69, 214202 (2004).

23. Zunger, A., Wei, S.-H., Ferreira, L. G. \& Bernard, J. E. Special quasirandom structures. Phys. Rev. Lett. 65, 353-356 (1990).

24. Wei, S.-H., Ferreira, L. G., Bernard, J. E. \& Zunger, A. Electronic properties of random alloys: special quasirandom structures. Phys. Rev. B 42, 9622-9649 (1990).

25. Hass, K. C., Davis, L. C. \& Zunger, A. Electronic structure of random $\mathrm{Al}_{0.5} \mathrm{Ga}_{0.5} \mathrm{As}$ alloys: test of the "special-quasirandom-structures" description. Phys. Rev. B 42, 3757-3760 (1990)

26. van de Walle, A., Asta, M. \& Ceder, G. The alloy theoretic automated toolkit: a user guide. Calphad 26, 539-553 (2002).

27. Hafner, J. Ab-initio simulations of materials using VASP: density-functional theory and beyond. J. Comput. Chem. 29, 2044-2078 (2008).

28. Mermin, N. D. Thermal properties of the inhomogeneous electron gas. Phys. Rev. 137, A1441-A1443 (1965).

29. Kresse, G. \& Joubert, D. From ultrasoft pseudopotentials to the projector augmented-wave method. Phys. Rev. B 59, 1758-1775 (1999).

30. Perdew, J. P., Burke, K. \& Ernzerhof, M. Generalized gradient approximation made simple. Phys. Rev. Lett. 77, 3865-3868 (1996).

31. Methfessel, M. \& Paxton, A. T. High-precision sampling for Brillouin-zone integration in metals. Phys. Rev. B 40, 3616-3621 (1989).

32. Monkhorst, H. J. \& Pack, J. D. Special points for Brillouin-zone integrations. Phys. Rev. B 13, 5188-5192 (1976).

33. Santos-Carballal, D., Roldan, A., Grau-Crespo, R. \& de Leeuw, N. H. A DFT study of the structures, stabilities and redox behaviour of the major surfaces of magnetite Fe304. Phys. Chem. Chem. Phys. 16, 21082-21097 (2014).

34. Haynes, W. M. CRC Handbook of Chemistry and Physics. (Boca Raton CRC Press, FL 2012).

35. Reuter, K. \& Scheffler, M. First-principles atomistic thermodynamics for oxidation catalysis: Surface phase diagrams and catalytically interesting regions. Phys. Rev. Lett. 90, 461031-461034 (2003).

36. Tang, W., Sanville, E. \& Henkelman, G. A grid-based Bader analysis algorithm without lattice bias. J. Phys. Condens. Matter 21, 084204 (2009).

Open Access This article is licensed under a Creative Commons Attribution 4.0 International License, which permits use, sharing, adaptation, distribution and reproduction in any medium or format, as long as you give appropriate credit to the original author(s) and the source, provide a link to the Creative Commons license, and indicate if changes were made. The images or other third party material in this article are included in the article's Creative Commons license, unless indicated otherwise in a credit line to the material. If material is not included in the article's Creative Commons license and your intended use is not permitted by statutory regulation or exceeds the permitted use, you will need to obtain permission directly from the copyright holder. To view a copy of this license, visit http://creativecommons. org/licenses/by/4.0/.

(c) The Author(s) 2019 